\title{
Prevalence of malnutrition and risk of undernutrition in hospitalised children with liver disease
}

\author{
Ronghua $\mathrm{Yu}^{1} \dagger$, Yizhong $\mathrm{Wang}^{1} \dagger$, Yongmei Xiao ${ }^{1}$, Lili Mo ${ }^{1}$, Aishu Liu ${ }^{1}$, Dan Li ${ }^{1}$, Ting $\mathrm{Ge}^{1}$, Guangjun $\mathrm{Yu}^{2} *$ \\ and Ting Zhang ${ }^{*}$ \\ ${ }^{1}$ Department of Gastroenterology, Hepatology, and Nutrition, Shanghai Children's Hospital, Shanghai Jiao Tong University, Shanghai 200062, \\ People's Republic of China \\ ${ }^{2}$ Department of Children's Healthcare, Shanghai Children's Hospital, Shanghai Jiao Tong University, Shanghai 200062, People's Republic of China
}

(Received 24 May 2017 - Final revision received 4 September 2017 - Accepted 14 September 2017)

Journal of Nutritional Science (2017), vol. 6, e55, page 1 of 5

doi:10.1017/jns.2017.56

\section{Abstract}

Nutritional status of 380 hospitalised children aged from 1 month to 5 years with liver disease was evaluated in a single paediatric centre. The total prevalence of stunting (height-for-age $Z$ (HAZ) $<-2$ ), underweight (weight-for-age $Z$ (WAZ) $<-2$ ) and wasting (weight-for-height $Z<-2$ ) was $9 \cdot 8,9 \cdot 0$ and $7.9 \%$, respectively. The overall nutritional risk $(-2 \leq Z<-1)$ of stunting, underweight and wasting was $11.8,12.9$ and $12.6 \%$. The prevalence of undernutrition was significantly higher in children with cholestasis than children without cholestasis (stunting, $17.5 \% / 4.4 \%, P<0.001$, and underweight, 14.9 $\% / 4.9 \%, P<0.001$ ). HAZ and WAZ scores were significantly higher in children without cholestasis than children with cholestasis $(0.58$ (SD 1.59$) /-0.68$ (sD 1.99), $P<0.001$, and 0.37 (SD 1.35)/ -0.47 (sD 1.75), $P<0.001$ ). Further multivariate logistic regression analysis strengthened the evidence that cholestasis was significantly associated with undernutrition of stunting $(\mathrm{OR}=4 \cdot 18, P=0 \cdot 002)$ and underweight $(\mathrm{OR}=3 \cdot 26, P=0 \cdot 008)$, and suggested that the prevalence of stunting caused by infection was lower than other aetiologies in hospitalised children with liver disease $(\mathrm{OR}=0 \cdot 10, P=0 \cdot 002)$. We concluded that a high prevalence of malnutrition and risk of undernutrition presents in hospitalised young children with liver disease, especially in children with cholestasis. Nutrition assessment is recommended for hospitalised children with liver disease.

\section{Key words: Liver disease: Children: Malnutrition: Undernutrition}

Malnutrition is a state of nutrition in which a deficiency or excess, or imbalance of energy, protein and other nutrients causes measurable adverse effects on tissue/body form, body function and clinical outcome ${ }^{(1)}$. The human liver is an important regulator of metabolism, storage, synthesis and absorption of nutrients ${ }^{(2)}$. Nutritional deficiencies are common among people with chronic liver disease, especially in children ${ }^{(3-5)}$. The malnutrition prevalence in children with chronic liver disease may range from $9 \cdot 1$ to $71.1 \%$ depending on the severity of liver disease ${ }^{(5-7)}$. The development of malnutrition in childhood liver disease is complex and involves multiple mechanisms, including decreased dietary intake, impaired nutrient digestion and absorption, increased energy requirements and disordered substrate use ${ }^{(8-10)}$. Liver disease patients with malnutrition have greater incidence of complications ${ }^{(11,12)}$. They have higher risk of infections, longer hospital stay and cost, and increased mortality after liver transplantation compared with well-nourished patients ${ }^{(13-16)}$. In addition, patients' nutrition status is one of the effective factors on the prognostic variables during liver transplantation ${ }^{(16,17)}$. Therefore, it is very important to assess the nutrition status of paediatric patients with chronic disease, including chronic liver disease.

Abbreviations: ALT, alanine transaminase; HAZ, height-for-age Z-score; WAZ, weight-for-age Z-score; WHZ, weight-for-height Z-score.

* Corresponding authors: T. Zhang and G. Yu, fax +86 21 52976338, email zhangt@shchildren.com.cn and gjyu@shchildren.com.cn

† Both authors contributed equally to this study. 
Currently, there are limited studies focused on the nutrition status of children with liver disease in China. The present study assessed the nutritional status, and evaluated the prevalence of malnutrition and risk of undernutrition of hospitalised children with liver disease at the Shanghai Children's Hospital.

\section{Methods}

\section{Study population}

This single-centre, cross-sectional prospective study was performed at the Department of Gastroenterology, Hepatology, and Nutrition, the Shanghai Children's Hospital, China. Patients were recruited from December 2013 to March 2016. Hospitalised patients, aged from 1 month to 5 years, with a diagnosis of liver disease were recruited in the study period. The diagnosis of liver disease is defined as satisfying at least one of the following clinical manifestations: hepatomegaly, jaundice and alanine transaminase (ALT) $>80 \mathrm{U} / 1$. This study was conducted according to the guidelines laid down in the Declaration of Helsinki and all procedures involving human subjects were approved by the Shanghai Children's Hospital Institutional Review Board. Written informed consent was obtained from all the guardians of participants.

\section{Data collection}

The data from patients' electronic medical records were collected, including demographics (age, sex) and clinical data (laboratory values and imaging study results). According to the recommendations of the North American Society for Pediatric Gastroenterology, Hepatology and Nutrition ${ }^{(18)}$, we defined an abnormal direct bilirubin as a value greater than $17 \cdot 1 \mu \mathrm{mol} / 1$ if the total bilirubin is less than $85.5 \mu \mathrm{mol} / 1$, or a value of direct bilirubin that represents more than $20 \%$ of the total bilirubin if the total bilirubin is greater than $85.5 \mu \mathrm{mol} / \mathrm{l}$. In the present study, the patients were divided into the cholestasis group and the non-cholestasis group according to the direct bilirubin value.

Anthropometric measures were completed within $24 \mathrm{~h}$ of admission, including weight and length/height. Children younger than 3 years and weighing up to $10 \mathrm{~kg}$ were measured in an electronic baby scale (SECA 335) without clothes and nappy; an electronic personal scale (SECA 704s) was used to measure children older than 3 years. Patients were evaluated barefoot and, usually, only with underwear. The equipment was checked before use. Data were analysed using the software Epi info version 3.5.4 (Centers for Disease Control and Prevention), and converted to the following variables: height-for-age $Z$-score (HAZ), weight-for-age $Z$-score (WAZ) and weight-for-height Z-score (WHZ). Nutrition risk and malnutrition were defined according to $Z$-score (nutrition risk: $-2 \leq Z<-1$; moderate malnutrition: $-3 \leq Z<-2$; severe malnutrition: $Z<-3$ ), while stunting, underweight and wasting were defined according to HAZ $(\mathrm{HAZ}<-2)$, WAZ (WAZ $<-2)$ and WHZ (WHZ $<-2)$, respectively.

\section{Statistical analysis}

Statistical analysis was performed by SPSS 20.0 software. The data are shown as medians and interquartile ranges (25th-75th percentile), or means with standard deviations, and compared using the non-parametric Mann-Whitney test. The distribution of dichotomous variables was compared using the $\chi^{2}$ test. The logistic regression model was generated using variables including sex, age, ALT $>80 \mathrm{U} / 1$, cholestasis and aetiology, in which aetiology was a categorical variable. The method of logistic regression model was 'enter'. A $P$ value of $<0.05$ was considered to be statistically significant.

\section{Results}

\section{Clinical characteristics}

A total of 380 hospitalised children with liver disease were evaluated, and their main clinical characteristics are shown in Table 1 . The median age was 5 months (interquartile range 2-20 months), and 59.5\% (226/380) of them were male. As for the age distribution, infants, toddlers and preschool children accounted for $67 \cdot 1 \%(255 / 380), 19 \cdot 2 \%(73 / 380)$ and $13.7 \%$ (52/380), respectively. In 380 children, 154 $(40.5 \%)$ subjects had cholestasis, $213(56.1 \%)$ had hepatomegaly, 191 (50.3\%) subjects had jaundice, and 262 (68.9

$\%$ ) cases had ALT more than $80 \mathrm{U} / 1$. The aetiologies of liver disease were: biliary atresia in twenty-four patients $(6 \cdot 3$ $\%$, Alagille syndrome in six $(1.6 \%)$, progressive familial intrahepatic cholestasis in eight $(2 \cdot 1 \%)$, glycogen storage disease in eight $(2.1 \%)$, Wilson disease in five $(1.3 \%)$, neonatal intrahepatic cholestasis in seven $(1.8 \%)$, viruses and other infectious hepatitis in $121(31.8 \%)$, and other diagnoses in thirty-four $(8.9 \%)$. The aetiologies of $167(43.9 \%)$ cases were undefined according to the medical record.

\section{Prevalence of nutritional risk and malnutrition}

As shown in Table 2 , the nutritional risk $(-2 \leq Z<-1)$ of stunting, underweight and wasting was $11.8 \%(45 / 380)$, $12.9 \%(49 / 380)$ and $12.6 \%(48 / 380)$, respectively. The total malnutrition prevalence of stunting (HAZ $<-2)$, underweight (WAZ $<-2$ ) and wasting (WHZ $<-2$ ) was $9.8 \%$ (37/380), $9.0 \%$ (34/380) and 7.9\% (30/380). Among the malnutrition patients, moderate malnutrition $(-3 \leq Z<-2)$ of stunting, underweight and wasting was observed in seventeen $(4.5 \%, 17 / 380)$, twenty $(5.3 \%, 20 / 380)$ and sixteen $(4.2 \%, 16 / 380)$ patients, while severe malnutrition $(Z<-3)$ was found in twenty $(5.3 \%, 20 / 380)$, fourteen $(3.7 \%, 14 /$ $380)$ and fourteen $(3.7 \%, 14 / 380)$ subjects, respectively.

\section{Factors associated with nutritional risk and malnutrition}

The 380 patients were divided into the cholestasis group and the non-cholestasis group according to the definition of cholestasis. The prevalence of stunting, underweight and wasting in children with cholestasis was $17.5 \%$ (27/154), $14.9 \%$ $(23 / 154)$ and $7.9 \%(13 / 152)$, while the prevalence of stunting, underweight and wasting in children without cholestasis 
Table 1. Clinical characteristics of 380 hospitalised children with liver disease (Mean values and interquartile ranges (IQR); numbers of children and percentages)

\begin{tabular}{|c|c|c|c|c|}
\hline Characteristics & Mean & IQR & $n$ & $\%$ \\
\hline \multicolumn{5}{|l|}{ Sex } \\
\hline Male & & & 266 & 59.5 \\
\hline Female & & & 154 & 40.5 \\
\hline Age (months) & 5 & 2,20 & & \\
\hline Hepatomegaly & & & 213 & $56 \cdot 1$ \\
\hline Cholestasis & & & 154 & $40 \cdot 5$ \\
\hline $\operatorname{ALT}(U / l)$ & 133 & 64,255 & & \\
\hline Total bilirubin $(\mu \mathrm{mol} / \mathrm{l})$ & 18.52 & $7.90,129.43$ & & \\
\hline Direct bilirubin $(\mu \mathrm{mol} / \mathrm{l})$ & 6.00 & $1 \cdot 90,65 \cdot 10$ & & \\
\hline \multicolumn{5}{|l|}{ Aetiology } \\
\hline Structural abnormalities & & & 32 & 8.4 \\
\hline Biliary atresia & & & 26 & $6 \cdot 8$ \\
\hline Others & & & 6 & 1.6 \\
\hline Genetic/metabolic disorders & & & 49 & 12.9 \\
\hline Alagille syndrome & & & 7 & 1.8 \\
\hline Progressive familial intrahepatic cholestasis & & & 8 & $2 \cdot 1$ \\
\hline Glycogen storage disease & & & 8 & $2 \cdot 1$ \\
\hline Wilson disease & & & 6 & 1.6 \\
\hline Neonatal intrahepatic cholestasis & & & 8 & $2 \cdot 1$ \\
\hline Others & & & 12 & 3.2 \\
\hline Infectious diseases & & & 151 & 39.7 \\
\hline Cytomegalovirus & & & 54 & $14 \cdot 2$ \\
\hline Epstein-Barr virus & & & 51 & 13.4 \\
\hline Hepatitis B virus & & & 7 & 1.8 \\
\hline Others & & & 46 & $12 \cdot 1$ \\
\hline Others & & & 32 & 8.4 \\
\hline Unknown & & & 125 & 32.9 \\
\hline
\end{tabular}

$\mathrm{ALT}$, alanine transaminase.

was $4.4 \%(10 / 226), 4.9 \%(11 / 226)$ and $7.6 \%(17 / 223)$, respectively (Table 3). The differences in stunting and underweight between the cholestasis group and non-cholestasis group were statistically significant $(P<0 \cdot 001, P=0.001)$; however, there was no statistically significant difference between the two groups in terms of wasting $(P=0.745$; Table 3$)$. The HAZ, WAZ and WHZ scores of the whole study patients were 0.07 (SD 1.86), 0.03 (SD 1.58) and -0.08 (sD 1.57), respectively (Table 4). The $Z$-scores of HAZ, WAZ and WHZ of the cholestasis group were lower than the noncholestasis group, and the differences in HAZ (0.58 (SD $1.59) /-0.68$ (SD 1.99), $P<0.001)$ and WAZ $(0.37$ (SD 1.35)/ -0.47 (sD 1.75$), P<0.001$ ) were statistically significant, but there was no significant difference between the two group in terms of WHZ ( -0.05 (SD 1.50)/ -0.11 (sD 1.66), $P=0.722$; Table 4). Further multivariate logistic regression analysis strengthened the evidence that cholestasis was significantly associated with undernutrition of stunting $(\mathrm{OR}=4 \cdot 18, P=0.002)$ and underweight $(\mathrm{OR}=3 \cdot 26, P=0 \cdot 008)$, and suggested that the prevalence of stunting caused by infection was lower than other aetiologies in hospitalised children with liver disease $(\mathrm{OR}=0 \cdot 10, P=0.002)($ Table 5).

\section{Discussion}

Nutritional deficiencies are common in children with chronic liver disease, especially when cholestasis is present and its onset occurs in the first year of life. Therefore, nutritional evaluation of these children is essential. In the present study, in order to provide reference for rational application of nutritional support, we examined the nutritional status of hospitalised children under 5 years old with a diagnosis of liver disease in a single paediatric centre in China. Finally, 380 in-patients were recruited in the study period. Clinical characteristics showed that $40 \cdot 5 \%$ subjects had cholestasis, $56 \cdot 1 \%$ cases had hepatomegaly, $50 \cdot 3 \%$ subjects had jaundice, and 262 $(68.9 \%)$ cases had ALT more than $80 \mathrm{U} / 1$. The main aetiologies of liver disease were structural abnormalities, genetic/ metabolic disorders and infectious disease. Nutrition assessment results showed that the prevalence of stunting,

Table 2. Prevalence of nutritional risk and malnutrition of the patients with liver disease (Numbers of children and percentages)

\begin{tabular}{|c|c|c|c|c|c|c|}
\hline \multirow[b]{2}{*}{ Nutrition status } & \multicolumn{2}{|c|}{ Stunting } & \multicolumn{2}{|c|}{ Underweight } & \multicolumn{2}{|c|}{ Wasting } \\
\hline & $n$ & $\%$ & $n$ & $\%$ & $n$ & $\%$ \\
\hline Nutritional risk $(-2 \leq Z<-1)$ & 45 & 11.8 & 49 & $12 \cdot 9$ & 48 & 12.6 \\
\hline Moderate malnutrition $(-3 \leq Z<-2)$ & 17 & 4.5 & 20 & $5 \cdot 3$ & 16 & 4.2 \\
\hline Severe malnutrition $(Z<-3)$ & 20 & $5 \cdot 3$ & 14 & 3.7 & 14 & 3.7 \\
\hline Total & 82 & 21.6 & 83 & 21.9 & 78 & 20.5 \\
\hline
\end{tabular}


Table 3. Comparison of malnutrition prevalence in patients with and without cholestasis

(Numbers of children and percentages)

\begin{tabular}{|c|c|c|c|c|c|c|}
\hline & \multicolumn{2}{|c|}{ Stunting } & \multicolumn{2}{|c|}{ Underweight } & \multicolumn{2}{|c|}{ Wasting } \\
\hline & $n / N$ & $\%$ & $n / N$ & $\%$ & $n / N$ & $\%$ \\
\hline With cholestasis & $27 / 154$ & 17.5 & $23 / 154$ & 14.9 & $13 / 152$ & 8.6 \\
\hline Without cholestasis & $10 / 226$ & 4.4 & $11 / 226$ & 4.9 & $17 / 223$ & 7.6 \\
\hline$\chi^{2}$ & \multicolumn{2}{|c|}{17.91} & \multicolumn{2}{|c|}{11.40} & \multicolumn{2}{|c|}{0.11} \\
\hline$P^{\star}$ & \multicolumn{2}{|c|}{$<0.001$} & \multicolumn{2}{|c|}{0.001} & \multicolumn{2}{|c|}{0.745} \\
\hline
\end{tabular}

underweight and wasting was $9 \cdot 8,9 \cdot 0 \%$ and $7.9 \%$, respectively, which was greater than the overall malnutrition rate of paediatric in-patients in Shanghai (stunting $7.1 \%$, underweight $5.5 \%$ and wasting $5 \cdot 2 \%$, respectively) reported in $2007^{(19)}$, and much greater than the overall rate of malnutrition in children under 5 years of age in cities of China in 2002 (stunting $4.9 \%$, underweight $3.1 \%$ and wasting $1.8 \%$, respectively) ${ }^{(20)}$. Severe malnutrition in wasting was greater than that in paediatric hospitalised patients in Germany $(3.7 \% .1 .7 \%)^{(21)}$. It has been suggested that liver disease is more likely to cause malnutrition, especially severe malnutrition, than other diseases in children. However, the malnutrition prevalence was lower than in a recent study in Brazil (stunting 50.0\%, underweight $27.3 \%$ and wasting $11.1 \%$, respectively) ${ }^{(5)}$. The difference may be attributed to the course and severity of the liver disease.

To further investigate the factors associated with malnutrition and risk of undernutrition in children with liver disease, 380 patients were divided into a cholestasis group and a noncholestasis group. Although there was no significant difference between the cholestasis group and non-cholestasis group in terms of wasting, the prevalence of stunting and underweight in the cholestasis group was significantly higher than in the non-cholestasis group. However, the prevalence of stunting $(17.5 \% / 4.4 \%)$ and underweight $(14.9 \% / 4.9 \%)$ in the two groups was lower than that observed in an earlier study $(64.3 \% / 18.2 \%, 57.2 \% / 9.1 \%)^{(22)}$. Similarly, the prevalence of stunting $(17.5 \%)$, underweight $(14.9 \%)$ and wasting $(8.6$

$\%)$ in the cholestasis group was lower than in another similar
Table 4. Z-scores of the anthropometric results of the whole group of patients and stratified according to the presence of cholestasis (Z-scores and standard deviations)

\begin{tabular}{|c|c|c|c|c|c|c|}
\hline & \multicolumn{2}{|c|}{ HAZ } & \multicolumn{2}{|c|}{ WAZ } & \multicolumn{2}{|c|}{ WHZ } \\
\hline & $z$ & SD & $z$ & SD & $z$ & SD \\
\hline General (380 children) & 0.07 & 1.86 & 0.03 & 1.58 & -0.08 & 1.57 \\
\hline $\begin{array}{l}\text { With cholestasis (154 } \\
\text { children) }\end{array}$ & -0.68 & 1.99 & -0.47 & 1.75 & $-0.11^{*}$ & 1.66 \\
\hline $\begin{array}{l}\text { Without cholestasis } \\
\text { (226 children) }\end{array}$ & 0.58 & 1.59 & 0.37 & 1.35 & $-0.05^{\dagger}$ & 1.50 \\
\hline$P \ddagger$ & \multicolumn{2}{|c|}{$<0.001$} & \multicolumn{2}{|c|}{$<0.001$} & \multicolumn{2}{|c|}{0.722} \\
\hline
\end{tabular}

HAZ, height-for-age Z-score; WAZ, weight-for-age Z-score; WHZ, weight-for-height $Z$-score.

* 152 Children

†223 Children.

‡ Independent-samples $t$ test (cholestasis group and non-cholestasis group).

study in Brazil (stunting 30.8\%, underweight 33.0\% and wasting $12 \cdot 1 \%$, respectively $)^{(5)}$. The reason may be that we have different criteria for the inclusion of cholestasis. In addition to cholestasis, multivariate logistic regression analysis suggested that earlier age and hepatomegaly were independent factors that were significantly associated with nutritional risk of underweight in hospitalised children with liver disease.

The authors are aware of limitations of the present study. In this study, children with liver disease had a variety of causes, and in different course and severity of disease, while the nutritional assessment was performed in a single moment. Another limitation is that body weight can be overestimated in cases of visceromegaly, ascites and peripheral oedema, at least in part, so weight for age, weight for height and BMI may underestimate the degree of undernutrition in children with chronic liver disease. The European Society of Clinical Nutrition and Metabolism (ESPEN) 2006 guideline recommends using Subjective Global Assessment and anthropometry to identify patients with cirrhosis who are at risk of undernutrition ${ }^{(23)}$. Some studies suggest that triceps skinfold thickness, mid-arm circumference, arm muscle circumference, global assessment of nutritional status and handgrip strength are indices which can better reflect the nutritional risk and the severity of cirrhosis in patients with chronic liver disease ${ }^{(5,24,25)}$. Thus, it is necessary to include more sensitive parameters to better

Table 5. Multivariate logistic regression analysis of variables associated with undernutrition (Odds ratios and $95 \%$ confidence intervals)

\begin{tabular}{|c|c|c|c|c|c|c|c|c|c|}
\hline \multirow[t]{2}{*}{ Variable } & \multicolumn{3}{|c|}{ Stunting } & \multicolumn{3}{|c|}{ Underweight } & \multicolumn{3}{|c|}{ Wasting } \\
\hline & OR & $95 \% \mathrm{Cl}$ & $P$ & OR & $95 \% \mathrm{Cl}$ & $P$ & OR & $95 \% \mathrm{Cl}$ & $P$ \\
\hline Male (yes/no) & 0.95 & $0.46,1.98$ & 0.899 & 1.34 & $0.62,2.88$ & 0.459 & 0.86 & $0.40,1.86$ & 0.701 \\
\hline Age (continuous) & 1.02 & $0.99,1.04$ & 0.211 & 1.00 & $0.98,1.03$ & 0.812 & 1.01 & $0.99,1.03$ & 0.338 \\
\hline ALT > 80 U/I (yes/no) & 0.69 & $0.32,1.46$ & 0.327 & 0.96 & $0.43,2.12$ & 0.915 & 1.77 & $0.35,1.86$ & 0.505 \\
\hline Cholestasis (yes/no) & $4 \cdot 18$ & $1 \cdot 72,10 \cdot 15$ & 0.002 & 3.26 & $1.36,7.79$ & 0.008 & 1.32 & $0.52,3.32$ & 0.556 \\
\hline \multicolumn{10}{|l|}{ Aetiology* } \\
\hline Others (reference group) & 1 & & 0.006 & 1 & & 0.029 & 1 & & 0.248 \\
\hline Structural abnormalities & 0.37 & $0.10,1.39$ & 0.141 & 0.00 & 0.00 & 0.998 & 2.54 & $0.74,8.72$ & 0.140 \\
\hline Genetic/metabolic disorders & $1 \cdot 21$ & $0.50,2.95$ & 0.672 & 2.04 & $0.84,4.95$ & 0.116 & 0.57 & $0.11,2.84$ & 0.491 \\
\hline Infectious diseases & $0 \cdot 10$ & $0.02,0.44$ & 0.002 & 0.35 & $0.12,1.01$ & 0.052 & 1.58 & $0.63,3.98$ & 0.331 \\
\hline
\end{tabular}

ALT, alanine transaminase.

${ }^{\star}$ Aetiology: 1, structural abnormalities; 2, genetic/metabolic disorders; 3, infectious diseases; 4, others. 
evaluate the nutritional status of children with liver disease in the future.

In conclusion, the malnutrition prevalence in hospitalised children with liver disease is high, especially in children with cholestasis. In order to shorten the hospitalisation time and improve the clinical outcome, nutrition assessment and timely nutritional support are recommended for hospitalised children with liver disease.

\section{Acknowledgements}

This work was supported by grants from the National Natural Science Foundation of China (Y. W., grant number 81500449) and the Natural Science Foundation of Shanghai (Y. W., grant number 14ZR1434200; T. Z., grant number 16ZR1428700).

Y. W., G. Y. and T. Z. were responsible for the conception and design of the work. R. Y., L. M. and A. L. acquired the data. R. Y., Y. X., D. L. and T. G. analysed and interpreted the data. R. Y., Y. W. and T. Z. drafted and revised the manuscript. All authors approved the final manuscript.

The authors have no conflicts of interest to disclose.

\section{References}

1. Remy Meier RS (2008) Basic concepts in nutrition: epidemiology of malnutrition. E Spen Eur E J Clin Nutr Metab 3, e167-e170.

2. Cheung K, Lee SS \& Raman M (2012) Prevalence and mechanisms of malnutrition in patients with advanced liver disease, and nutrition management strategies. Clin Gastroenterol Hepatol 10, 117-125.

3. Mager DR, McGee PL, Furuya KN, et al. (2006) Prevalence of vitamin $\mathrm{K}$ deficiency in children with mild to moderate chronic liver disease. J Pediatr Gastroenterol Nutr 42, 71-76.

4. Saron ML, Godoy HT \& Hessel G (2009) Nutritional status of patients with biliary atresia and autoimmune hepatitis related to serum levels of vitamins A, D and E. Arq Gastroenterol 46, 62-68.

5. da Silva FV, Ferri PM, Nascentes Queiroz TC, et al. (2016) Nutritional evaluation of children with chronic cholestatic disease. J Pediatr (Rio J) 92, 197-205.

6. Zamberlan P, Leone C, Tannuri U, et al. (2012) Nutritional risk and anthropometric evaluation in pediatric liver transplantation. Clinics (Sao Paulo) 67, 1387-1392.

7. Santetti D, de Albuquerque Wilasco MI, Dornelles CT, et al. (2015) Serum proinflammatory cytokines and nutritional status in pediatric chronic liver disease. World J Gastroenterol 21, 8927-8934.

8. Protheroe SM (1998) Feeding the child with chronic liver disease. Nutrition 14, 796-800.
9. Nightingale S \& Ng VL (2009) Optimizing nutritional management in children with chronic liver disease. Pediatr Clin North Am 56, 1161-1183.

10. Francavilla R, Miniello VL, Brunetti L, et al. (2003) Hepatitis and cholestasis in infancy: clinical and nutritional aspects. Acta Paediatr Suppl 91, 101-104.

11. Huisman EJ, Trip EJ, Siersema PD, et al. (2011) Protein energy malnutrition predicts complications in liver cirrhosis. Eur $J$ Gastroenterol Hepatol 23, 982-989.

12. Sam J \& Nguyen GC (2009) Protein-calorie malnutrition as a prognostic indicator of mortality among patients hospitalized with cirrhosis and portal hypertension. Liver Int 29, 1396-1402.

13. Merli M, Giusto M, Gentili F, et al. (2010) Nutritional status: its influence on the outcome of patients undergoing liver transplantation. Liver Int 30, 208-214.

14. Barshes NR, Chang IF, Karpen SJ, et al. (2006) Impact of pretransplant growth retardation in pediatric liver transplantation. J Pediatr Gastroenterol Nutr 43, 89-94.

15. Bucuvalas JC, Zeng L \& Anand R (2004) Predictors of length of stay for pediatric liver transplant recipients. Liver Transpl 10, 1011-1017.

16. Alonso EM, Martz K, Wang D, et al. (2013) Factors predicting health-related quality of life in pediatric liver transplant recipients in the functional outcomes group. Pediatr Transplant 17, 605-611.

17. Haseli N, Hassanzadeh J, Dehghani SM, et al. (2013) Long-term survival and its related factors in pediatric liver transplant recipients of Shiraz Transplant Center, Shiraz, Iran in 2012. Hepat Mon 13, e10257.

18. Moyer V, Freese DK, Whitington PF, et al. (2004) Guideline for the evaluation of cholestatic jaundice in infants: recommendations of the North American Society for Pediatric Gastroenterology, Hepatology and Nutrition. J Pediatr Gastroenterol Nutr 39, 115-128.

19. Tao Y-X, Xu Y-F, Tang Q-Y, et al. (2007) Nutrition assessment in hospitalized children [in Chinese]. Chin J Clin Nutr 15, 214-217.

20. Li LM, Rao KQ, Kong LZ, et al. (2005) [A description on the Chinese National Nutrition and Health Survey in 2002]. Zhonghua Liu Xing Bing Xue Za Zhi 26, 478-484.

21. Pawellek I, Dokoupil K \& Koletzko B (2008) Prevalence of malnutrition in paediatric hospital patients. Clin Nutr 27, 72-76.

22. Mattar RH, Azevedo RA, Speridiao PG, et al. (2005) [Nutritional status and intestinal iron absorption in children with chronic hepatic disease with and without cholestasis]. J Pediatr (Rio J) 81, 317-324.

23. Plauth M, Cabre E, Riggio O, et al. (2006) ESPEN guidelines on enteral nutrition: liver disease. Clin Nutr 25, 285-294.

24. Houissa F, Salem M, Debbeche R, et al. (2010) Evaluation of nutritional status in patients with liver cirrhosis. Tunis Med 88, 76-79.

25. Alvares-da-Silva MR \& Reverbel da Silveira T (2005) Comparison between handgrip strength, subjective global assessment, and prognostic nutritional index in assessing malnutrition and predicting clinical outcome in cirrhotic outpatients. Nutrition 21, 113-117. 